\title{
A GÊNESE CLIMÁTICA DOS TORNADOS NOS ESTADOS DA REGIÃO SUL DO BRASIL E SÃO PAULO
}

\author{
THE CLIMATIC GENESIS OF TORNADOES IN THE STATES OF THE SOUTHERN REGION OF BRAZIL AND SÃO \\ PAULO
}

\author{
Cássio Arthur Wollmann¹, Amanda Comassetto lensse ${ }^{1}$ \\ 1 Universidade Federal de Santa Maria (UFSM), Santa Maria, RS, Brasil.
}

Correspondência para: Cássio Arthur Wollmann (cassio_geo@yahoo.com.br)

doi: 10.12957/geouerj.2019.40941

Recebido em: 31jul.2018 | Aceito em: 23 out. 2018

\section{RESUMO}

Os tornados são eventos atmosféricos intensos e extremos, sendo um dos desastres naturais de maior dificuldade de previsão. Assim sendo, esta pesquisa teve como objetivo principal analisar a circulação atmosférica regional envolvida na gênese deste fenômeno na Região Sul do Brasil e no estado de São Paulo. A metodologia utilizada baseou-se em revisão bibliográfica, descrição da área de estudo, construção de banco de dados de registros de tornados entre 1959 a 2018, e na utilização de Cartas Sinóticas da Marinha do Brasil e Imagens de Satélite do INPE, no dia de cada evento para identificação do sistema atmosférico atuante. A análise do banco de dados demonstrou um aumento no número de registros após a década de 1990, com pico entre 2006 e 2009. Conclui-se que, o sistema atmosférico de maior participação na gênese dos eventos tornádicos para a área de estudo foi a Frente Polar Atlântica, participando em 38,4\% de todos os 138 registros de tornados. Por fim, a análise da sazonalidade dos registros de tornados evidenciou que os eventos ocorrem principalmente nos meses da primavera e verão, totalizando juntos 59,4 \% dos casos.

Palavras-chave: Tornados. sistemas atmosféricos. Região Sul. São Paulo. sazonalidade.

\section{ABSTRACT}

Tornadoes are intense and extreme atmospheric events, being one of the most difficult natural disasters with anticipating forecasting. So, this research concerned in to analyze the regional atmospheric circulation involved in the genesis of this phenomenon in the Southern Region of Brazil and in the state of São Paulo. The methodology used was based on bibliographic review, description of the study area, construction of a database of tornado records between 1959 and 2018, and the use of synoptic charts of the Brazilian Navy and INPE satellite images, on the day of each event to identify the active atmospheric system. The analysis of the database showed an increase in the number of records after the 1990s, with a peak between 2006 and 2009. It can be conclude that the atmospheric system with the greatest participation in the genesis of tornadic events for the study area was the Atlantic Polar Front, accounting for $38.4 \%$ of all 138 tornadoes records. Finally, the analysis of the seasonality of tornadoes records showed that events occur mainly in the spring and summer months, totaling $59.4 \%$ of the cases together.

Keywords: Tornadoes. atmospheric systems. Southern Region. São Paulo. seasonality

\section{INTRODUÇÃO}

A crença de que os tornados só ocorriam na América do Norte, no Corredor dos Tornados, tem sido desmistificada nos últimos anos, com realização de pesquisas em diversas partes do mundo acerca dos eventos tornádicos. Apesar dos Estados Unidos registrarem o maior número de tornados tocando o 
solo anualmente, os tornados já foram testemunhados em todos os continentes, exceto Antártida (NOAA, 2018a).

A dinâmica da atmosfera faz parte das manifestações da natureza e ocorrerá com ou sem a presença do homem, e suas consequências podem variar. A preocupação maior acerca desses fenômenos naturais é quando resulta em vítimas fatais, e com o aumento da população, seja urbana ou rural, tais eventos acabam por serem registrados mais frequentemente (RODRIGUES, 2011).

Os tornados são fenômenos atmosféricos extremos e intensos, e quando atingem alguma área habitada, ou com certo valor econômico, causam grandes transtornos e em casos mais extremos, vítimas. Tornados representam ameaça potencial para atividades importantes como aviação, agricultura, transmissão e distribuição de energia elétrica (NASCIMENTO, 2005).

Durante muitos anos, a definição mais amplamente aceita de um tornado foi encontrada, entre outras fontes, no Glossário de Meteorologia de Huschke (1959), caracterizando um tornado como uma coluna de ar de rotação violenta, pendente de uma nuvem de cumulonimbus, e quase sempre observável como uma "nuvem de funil". Porém essa edição não falava sobre a entrada da coluna de ar rotativa em contato com a superfície. No entanto, a próxima edição do Glossário de Glickman (2000) caracterizouo como uma coluna de ar que roda violentamente, em contato com o solo, pendente de uma nuvem cumulonimbus, ou embaixo de uma nuvem cumuliforme, e muitas vezes (mas nem sempre) é visível como uma nuvem de funil.

Na América do Sul, o corredor dos Tornados compreende países como Brasil, Argentina, Uruguai, Paraguai e Bolívia (VESILIND, 2004). A área selecionada para essa pesquisa no Brasil possui em sua gênese climática tanto sistemas atmosféricos tropicais quanto extratropicais, propiciando a formação de contrastes térmicos que geralmente promovem muita convecção atmosférica, levando à ocorrência de chuvas fortes, tempestades com granizo e eventualmente tornados (CANDIDO, 2012). 
Ainda, Candido (op. cit.) avaliou que os tornados surgem porque a turbulência aliada à redução da temperatura em certos pontos no interior das nuvens propicia ocorrência de ventos rotacionais que, em condições ideais, pouco estudadas, podem afunilar e, eventualmente, tocar o solo.

Além disso, entre 2001 e 2015 foram registrados 63 tornados só no estado do Rio Grande do Sul, de acordo com Iensse (2015). O crescimento no registro desses eventos deve-se principalmente ao fato do avanço tecnológico e ao crescimento da população. Entre os estudos que tratam de tornados no Brasil estão os publicados por Lima (1982), Silva Dias e Grammelsbacher (1991), Massambani, et. al. (1992), Nechet (2002), Reckziegel (2007), Rodrigues (2011), Candido (2012), Bertoni (2013) e Iensse (2015).

Com base na literatura estudada e com vistas ao preenchimento de lacunas do ponto de vista das análises climáticas aplicadas aos eventos tornádicos, o objetivo desta pesquisa consistiu em analisar os sistemas atmosféricos envolvidos na gênese deste fenômeno nos três estados da Região Sul do Brasil e no estado de São Paulo, realizando-se levantamento de registros, bem como se calculando sua sazonalidade.

Cabe destacar que sendo este estudo uma associação das condições atmosféricas de escala regional com um fenômeno que repercute em uma escala de dimensão espacial e temporal limitada, reforça-se que a questão escalar das condições atmosféricas prevalecentes em escala regional e a situação determinante para ocorrência do fenômeno em escala local refere-se aos condicionantes locais que são muito mais relevantes no advento desse tipo de perturbação, mas que não serão abordados nesta pesquisa.

\section{LOCALIZAÇÃO DA ÁREA DE ESTUDO}

Com o objetivo de analisar a circulação atmosférica envolvida nos registros de tornados e sua sazonalidade dos eventos na área de estudo, buscou-se dividir essa pesquisa em quatro etapas. A primeira etapa constituiu-se em revisão de bibliografia sobre os tornados além da circulação atmosférica do recorte espacial em questão. A segunda etapa estabeleceu-se a partir da localização e 
breve descrição da área de estudo. A terceira etapa foi de construção do banco de dados de tornados e sistemas atmosféricos e a quarta etapa foi de análise atmosférica em relação aos registros de tornados e sua contabilização sazonal.

A área de estudo (Figura 1) compreende os estados do Sul do Brasil (Paraná, Santa Catarina e Rio Grande do Sul) e um estado da região Sudeste (São Paulo), estados com o maior número de registros no território brasileiro, segundo Rodrigues (2011).

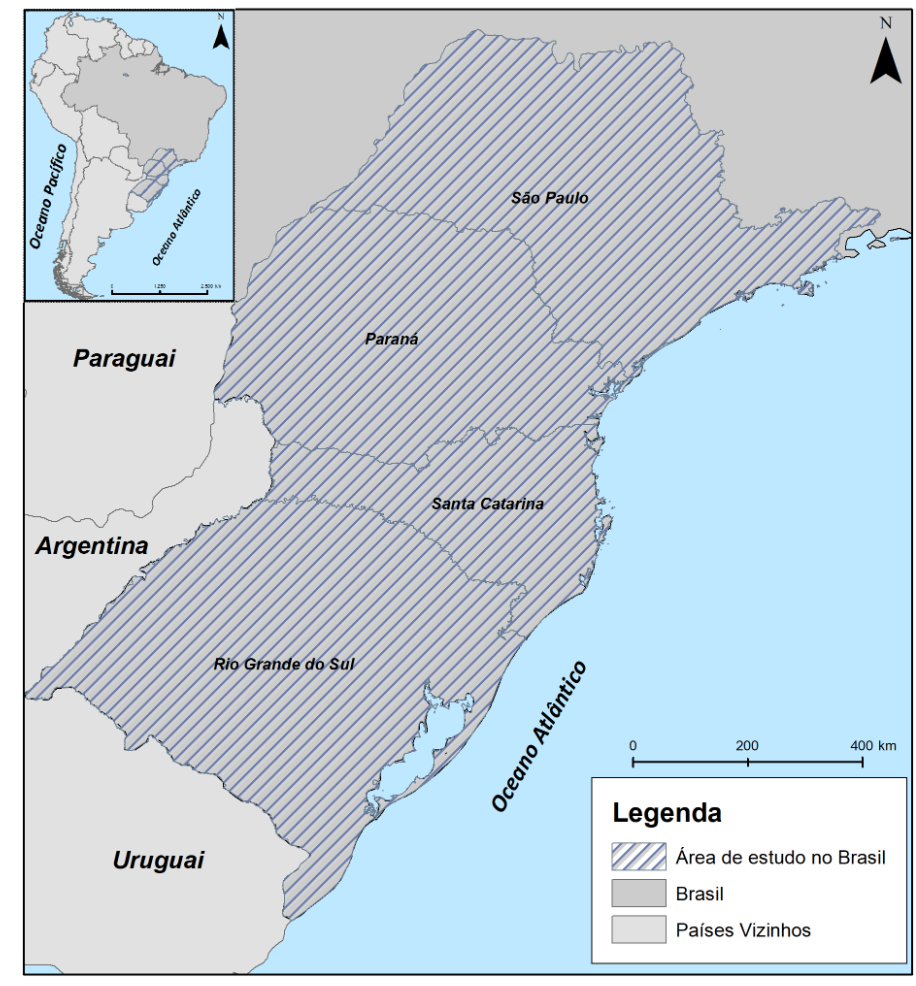

Figura 1. Localização da área de estudo em relação à América do Sul. Fonte: Os Autores

A hidrografia da área de estudo faz parte do sistema fluvial das bacias do Atlântico Sul e Sudeste, além do Rio da Prata e se estende por cinco países da América do Sul. Com uma extensão de $3.107 .000 \mathrm{~km}^{2}$ o sistema constitui-se por três grandes unidades hidrográficas, sendo elas: Rios Paraná, Paraguai e Uruguai (IENSSE, 2015). Em relação à hipsometria do terreno na área de estudo, nota-se que $70 \%$ encontra-se entre $50 \mathrm{~m}$ e $200 \mathrm{~m}$ de altitude e $30 \%$ da área de estudo possuem altitudes maiores que 200 m ficando restrita a Serra Geral, Serra do Mar, Serra da Mantiqueira e Planalto Meridional, no Brasil (Figura 2). 


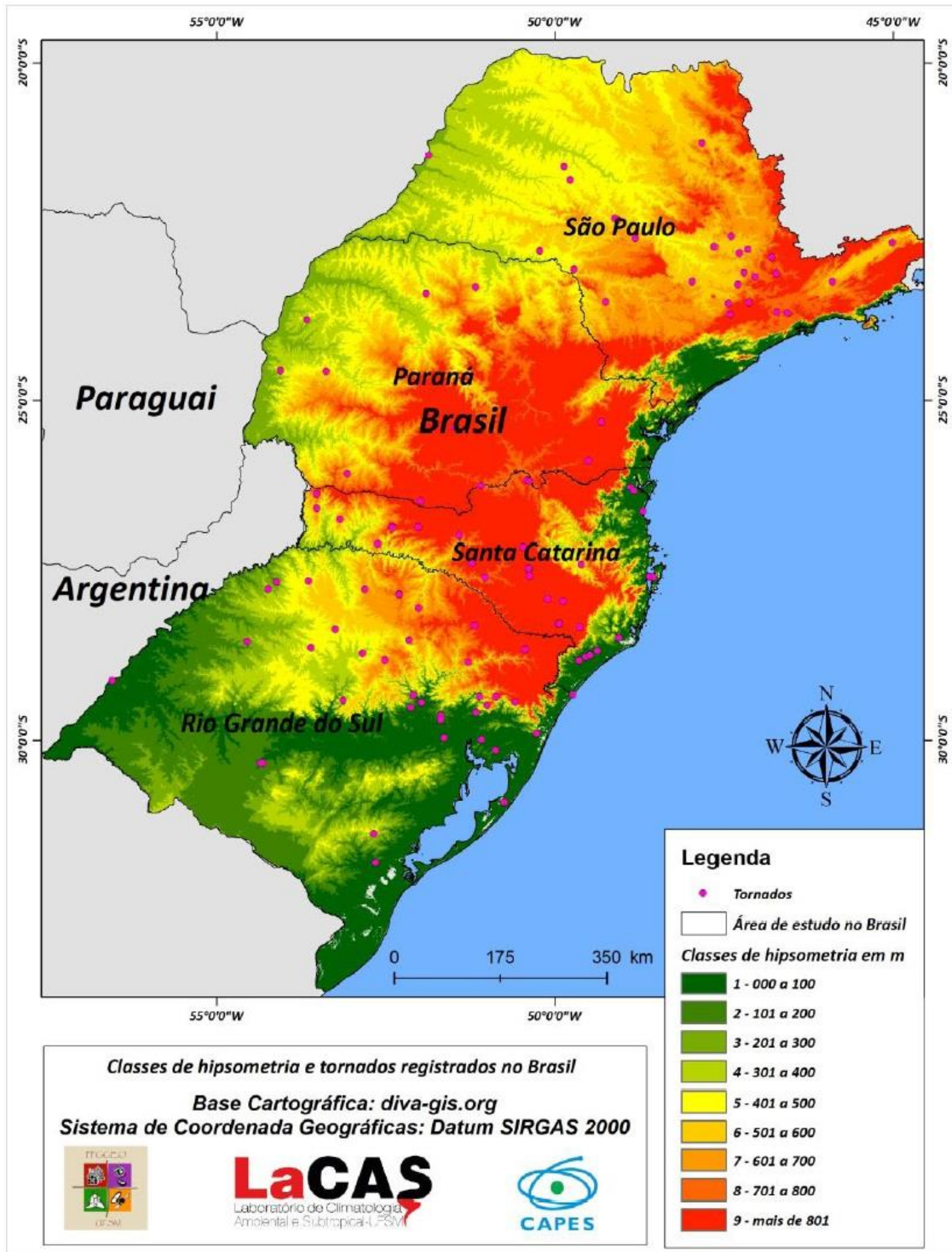

Figura 2. Hipsometria da área de estudo e localização dos registros de tornados conformes as classes de altitude adotadas.

Fonte: Os Autores

Ao observar a dinâmica atmosférica regional da área de estudo, nota-se semelhança com o Corredor dos Tornados dos Estados Unidos, em especial, ao observar-se que o ar frio vindo das latitudes subpolares em ambos os hemisférios, tende a encontrar-se com o ar quente e seco continental, e que no 
caso da América do Sul, trata-se da massa de ar originada na Baixa do Chaco e que se origina a leste do Andes pelo extravasamento da Massa Polar Pacífica sobre a cordilheira, e sofre dissecação adiabática (NIMER, 1989).

Observa-se também o ar quente e úmido vindo do oceano originado da Massa Tropical Atlântida além da corrente do Jato que atua em alto nível da atmosfera se deslocando de oeste para leste, colaborando para trocas de energia na atmosfera (GUEDES, 1985; VELASCO e FRITSCH, 1987). Todos os sistemas atmosféricos descritos são elementos que favorecem as condições que eventualmente podem formar tempestades tornádicas. Nesse contexto, a Figura 3 mostra o Corredor dos Tornados da América do Sul e os sistemas que compõem a atmosfera da área de estudo. 0 tamanho dos tornados não obedece a escala do mapa, e possuem meramente aspecto ilustrativo.

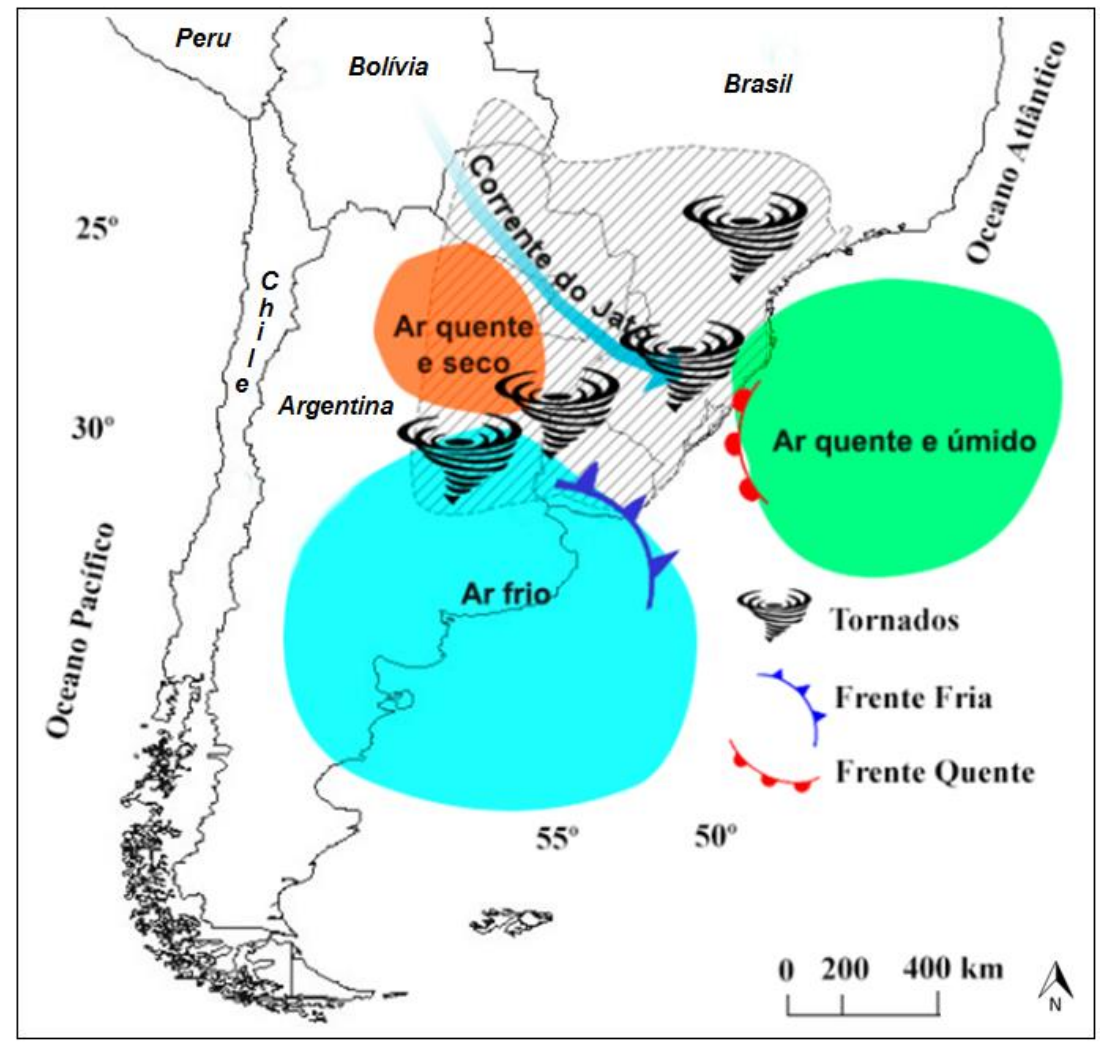

Figura 3. Corredor dos tornados da América do Sul e seus sistemas atmosféricos. Org.: Os autores (2018).

\section{MATERIAIS E MÉTODOS}

\section{Construção do Banco de dados}


De acordo com Chaudhuri (1998) as análises dos comportamentos dos dados geram informações extremamente úteis para tomada de decisões. Os dados podem ou não gerar informações dependendo da forma em que for manipulado, o uso de técnicas e ferramentas para analisar de forma inteligente tais dados é necessário para extrair informações e aprimorar o conhecimento (FAYYAD, 1996).

O banco de dados dos tornados foi construído através da coleta de dados divulgados e confirmados por páginas da web como $\mathrm{Metsul}^{1}$, Climatempo ${ }^{2}$ e do Instituto Nacional de Pesquisas Espaciais (INPE) ${ }^{3}$, trabalhos acadêmicos publicados e Defesa Civil dos quatro estados estudados: Paraná, Rio Grande do Sul, Santa Catarina e São Paulo. Buscaram-se também notícias de jornais locais de alguns registros incertos para uma melhor fidelidade, além de vídeos no site Youtube que mostram o evento e a data com vistas à confirmação do evento tornádico ${ }^{4}$.

\section{Análise atmosférica}

O levantamento de dados sobre a gênese dos tornados na área de estudo foi feito a partir de registros dos tornados confirmados. Com a coleta dos dados dos tornados, foi possível fazer a exclusão dos registros anteriores a 1959, pois não havia dados de cartas sinóticas e/ou imagens de satélite para a análise da circulação atmosférica anterior a essa data. Para a análise dos sistemas atmosféricos, foram utilizadas as Cartas Sinóticas das 12h e 00h do dia de cada evento, contabilizando 138 eventos.

O período de análise dos sistemas atmosféricos foi de 60 anos (1959 a 2018) e a partir de tabelas criadas em Excel, plotaram-se o local do evento, a data, o sistema atmosférico atuante no dia de registro e a sazonalidade, adotando, verão (Dezembro/Janeiro/Fevereiro - DJF), outono (Março/Abril/Maio - MAM), inverno (Junho/julho/Agosto - JJA) e primavera (Setembro/Outubro/Novembro - SON), segundo critério de Sartori (2003).

\footnotetext{
${ }^{1}$ www.metsul.com/

${ }^{2}$ www.climatempo.com.br/

${ }^{3}$ www.inpe.br/

${ }^{4}$ Localização dos eventos no mapa da Figura 02.
} 


\section{RESULTADOS E DISCUSSÕES}

No Brasil, os registros de tornados ocorridos entre 1959 a 2018 (Figura 4) somaram 138 eventos.Entre os anos de 1959 a 1990 os registros de tornados ainda eram escassos, pois dificilmente havia uma testemunha ocular ciente de tal evento que pudesse registrá-lo com um equipamento de captura de imagem, ou ainda pela falta de população nos locais de ocorrência.

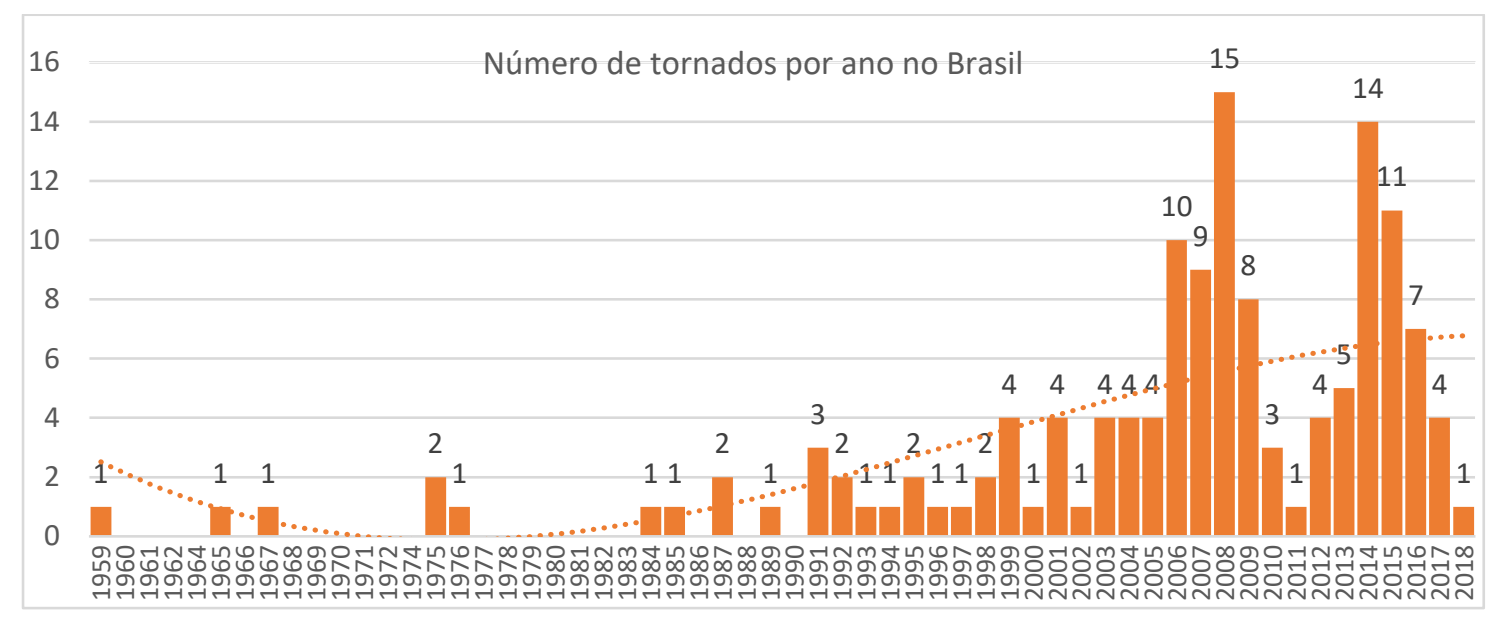

Figura 4. Número de tornados registrados na área de estudo entre 1959 e 2018.Org.: Os autores (2018).

Nos Estados Unidos, segundo NOAA (1995), houve um expressivo número de ocorrência de tornados entre os dias 03 e 04 de abril de 1974, quando foram registrados mais de 100 tornados somente nos dois dias. O fato, que foi chamado de "superoutbreak", tem relação com o avanço tecnológico na detecção e monitoramento de tais eventos climáticos nos Estados Unidos. No caso do Brasil, antes dos anos de 1990, frisa-se que o primeiro registro fotográfico oficialmente publicado do fenômeno foi registrado em 1975 em Santa Maria, no Rio Grande do Sul (LIMA, 1982).A distribuição anual dos tornados na área de estudo mostrou tendência de aumento entre os anos 2006 e 2009, com 42 tornados registrados $(30,4 \%$ dos registros em apenas quatro anos). Salienta-se que em 2006, o Brasil encontrava-se sob influência de La Niña fraca (NOAA, 2018b).

Iensse (2015) ao associar os tornados no Rio Grande do Sul com os fenômenos ENOS notou grande tendência de os eventos acontecerem em situações de La Niña e posteriormente em anos neutros. Infere-se que essa situação seja causada pela diminuição da frequência de ocorrência das correntes 
perturbadas, situação esta que leva a uma diminuição da nebulosidade e maior entrada de radiação solar, que promoverá a convecção e podendo favorecer a ocorrência de tornados.

Posteriormente os registros aumentaram somente no ano de 2014, com 14 tornados nesse ano e totalizando $10,1 \%$ dos registros. Nos demais anos, não há nenhuma tendência expressiva de aumento ou diminuição dos registros de tornados; por outro lado, não se pode afirmar que não houve evento, mas apenas não houve o registro de evento. A Figura 5 apresenta a análise dos sistemas atmosféricos envolvidos na gênese dos registros de eventos tornádicos na área de estudo entre 1959 e 2018.

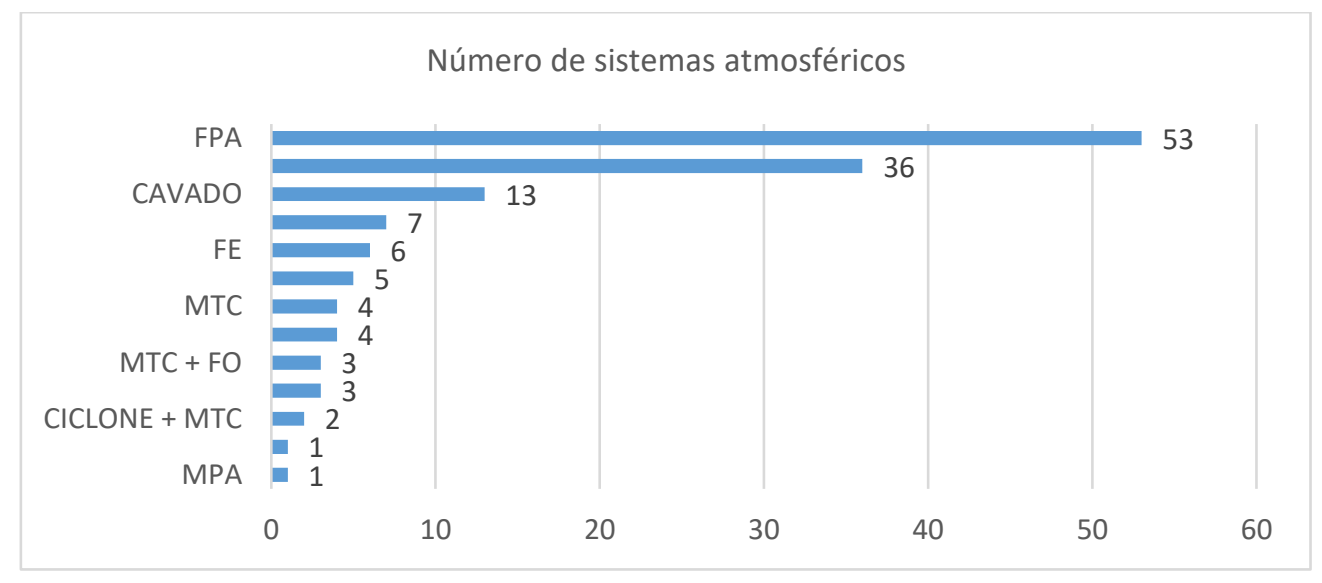

Figura 4. Total de tornados registrados por sistema atmosférico Org.: Os autores (2018).Legenda: FPA (Frente Polar Atlântica) MTC (Massa Tropical Continental) FE (Frente Estacionária) CCM (Complexo Convectivo de Mesoescala); FO (Frente Oclusa); MPA (Massa Polar Atlântica).

A FPA foi o sistema atmosférico que teve maior participação na área de estudo, totalizando 53 registros sob sua influência $(38,4 \%)$.A FPA possui em sua gênese a capacidade de produzir zonas de descontinuidades barométricas, térmicas, higrométricas e anemométricas, ou seja, a passagem de uma Frente por determinada região é acompanhada de instabilidade atmosférica (MENDONÇA e DANNIOLIVEIRA, 2007). Além disso, as frentes têm o papel de separar o ar polar e o ar tropical e participam ativamente da sucessão de tipos de tempo (SARTORI, 2003). As diferenças de temperaturas e pressão das massas de ar são muito acentuadas, proporcionando precipitação por vezes intensas devido às correntes convectivas e eventualmente podem formar tornados. 
A FPA ainda foi observada associada com cavado em três eventos, ou seja,2,2\% das participações, evidenciando sua importância no quadro geral da circulação atmosférica na área de estudo. Sartori (op. cit.) coloca que, no Rio Grande do Sul, a participação da FPA é maior no inverno,permanecendo22\% dos dias sobre a área de estudo, e ainda coloca que no verão a FPA aparece como o segundo sistema atmosférico de maior participação nos dias com $20 \%$ de participação.

Em segundo lugar, o sistema que teve mais participação foram os Ciclones Frontais, isto é, a FPA com ciclogênese, com a participação em 36 registros de eventos tornádicos (26,1\% dos registros). Por ser parte do sistema frontal, o ciclone possui sua gênese igualmente associada à FPA, porém seu desenvolvimento possui etapas bem definidas e são responsáveis por grandes índices pluviométricos de acordo com Sartori (2003).

É importante salientar que a escala climática a qual ocorre um Ciclone ou FPA é diferente da escala a qual o tornado vem a se desenvolver. A formação de um tornado está relacionada às instabilidades presente no local de ocorrência, assim como depende dos fatores geográficos presentes (RIBEIRO, 1993).

Os cavados estiveram presentes em 13registros $(9,4 \%)$ de tornados na área de estudo; esses sistemas em geral estão associados com a FPA e possuem a qualidade de provocar instabilidades severas. Cossetin, et. al. (2016) avaliaram dois eventos severos associados aos cavados no sul do Brasil, onde pelo menos 30 municípios registraram granizo e rajadas de ventos de mais de $100 \mathrm{~km} / \mathrm{h}$. Nesse sentido, a presença de cavados nos registros de tornados, torna-se um excelente indício para ser estudado individualmente, pois foi o terceiro sistema que teve mais participação no registro dos tornados.

A FPA associada à MTC esteve presente em sete registros $(5,1 \%)$ e posteriormente a MTC apareceu atuando majoritariamente na área de estudo em quatro registros (2,9\%), e ainda estando associada aos CCMs em outros cinco registros $(3,6 \%)$ e em dois registros associada ao Ciclone, contabilizando 1,4\% dos registros. Apesar de a MTC ser quente e seca por natureza, ela proporciona o aquecimento, 
estando presente nos dias mais quentes do ano. Por outro lado, se a MTC não possui umidade, ela tem a capacidade de atrair outros sistemas atmosféricos, pois sua gênese está no centro de baixa pressão da Baixa do Chaco (SARTORI, 2003).

De acordo com Sartori (op. cit.),ao atrair outros sistemas atmosféricos, a MTC interage com sistemas tropicais e extratropicais e acaba se tornando responsável pelas Instabilidades Tropicais e Calhas Induzidas. Esses sistemas estão intimamente relacionados a distúrbios baroclínicos, proporcionando cenário ideal para formações de nuvens cumuliformes de grande desenvolvimento vertical, e, consequentemente tornados.

A MPA, ainda esteve presente em um evento registrado, somando 0,7\% de participação. Apesar de ser uma massa de ar de alta pressão e consequentemente estável, ao se associar com um cavado ou outro sistema em níveis superiores na atmosfera ela pode gerar tempo severo, pois além de receber influência de níveis superiores, a MPA ainda recebe muita umidade durante seu deslocamento desde as latitudes subpolares até seu destino final em latitudes tropicais (GALVANI e AZEVEDO, 2012; NIMER, 1966).

A Frente Estacionária esteve presente em seis eventos, somando 4,3\% dos registros. Semelhante aos efeitos de uma FPA, a frente estacionária se diferencia pela velocidade a qual se desloca, sendo lenta ou quase estacionada. A FE acaba por promover instabilidades até sua dissipação, o que pode levar vários dias (SARTORI, 2003).

Ao final, observou-se que dos 138 eventos registrados, 104 deles $(75,4 \%)$ estiveram associados exclusivamente aos sistemas extratropicais. Outros 12 eventos $(8,7 \%$ dos registros) foram dados de sistemas extratropicais associados a sistemas intertropicais. Somente 22 eventos $(15,9 \%)$ tiveram sua gênese exclusivamente motivada por sistemas intertropicais. Sendo assim, observa-se o domínio dos sistemas extratropicais (massas e correntes perturbadas) em $84,1 \%$ dos eventos tornádicos registrados na área de estudo. 


\section{Sazonalidade dos eventos tornádicos}

O inverno possui uma característica peculiar, pois recebe menos incidência de raios solares, resfriando o continente. Dito isto, a dinâmica de massas de ar de maiores temperaturas, como é o caso da MTC, vinculada à baixa do Chaco, acaba por ter uma menor atuação. Sendo assim, as massas de origem polar (MPA) e Correntes perturbadas de sul (FPA) possuem uma maior participação nos tipos de tempo, especialmente no Rio Grande do Sul, de acordo com SARTORI (2003). Todavia, alerta-se que as condições não são idênticas nos outros estados avaliados nesta pesquisa. Ao todo ocorreram 22 $(15,9 \%)$ registros de tornados no inverno.

Por outro lado, após a passagem de uma FPA a MPA invade o continente, trazendo o ar polar, mas também promove a abertura do céu para incidência de raios solares e, consequentemente, 0 aquecimento do continente. Nessa dinâmica, na chegada de uma nova FPA, as instabilidades e distúrbios de pressão podem ocorrer, e dessa forma gerar eventos tornádicos, especialmente nos anos de La Niña, quando a dinâmica atmosférica da área de estudo torna-se desprovida da participação de sistemas frontais (IENSSE, 2015).

As estações de transição mostraram-se bem propícias para a formação de tornados na área de estudo, pois o outono registrou 34 dos eventos, somando $24,6 \%$. Nesta estação transicional entre o verão e 0 inverno que o confronto entre os sistemas tropicais e extratropicais é maior, e causam turbulências na atmosfera. Ainda se destacam o atraso do aquecimento dos oceanos que possuem influência no aumento da umidade atmosférica provocado pela evaporação, e que favorece às ciclogêneses.

A primavera, por sua vez, registrou 42 dos tornados contabilizando $30,4 \%$ de todos os registros e assim como o outono, é uma estação de transição quando o continente começa a receber maior incidência de raios solares. Com a chegada da primavera, a massas de ar de origem tropical passam a ter maior participação na área de estudo e com isso geram energia necessária para formação de tempo severo. 
No verão, a área de estudo registrou 40 tornados, isto é, $29,0 \%$ de todos os eventos. Esse fato pode estar ligado aos Complexos Convectivos de Mesoescala (CCM); de acordo com Guedes (1985), no verão esses sistemas se deslocam de sudoeste para nordeste dentro da área de estudo, e podem percorrer uma distância maior, quando comparada com o período de inverno.

A previsão dos CMM's é difícil, pois não estão associados a nenhum tipo de sistema meteorológico, como frentes frias ou linhas de instabilidade. Suas gêneses e desenvolvimentos são resultantes da umidade disponível na atmosfera, em baixos níveis; da circulação do ar superior, em altos níveis; e da circulação local (MADDOX, 1980). Esse cenário descrito por Maddox (op. cit.) é ideal para a criação de instabilidade o que pode resultar na formação de eventos tornádicos.

É importante salientar que esse tipo de sistema convectivo é comum nas regiões que compreendem a Argentina e o sul do Brasil, mais especificamente Rio Grande do Sul e Santa Catarina. Segundo Guedes (1985) e Velasco e Fritsch (1987), a principal característica desse fenômeno é a presença de um jato em baixos níveis, proveniente da região Amazônica, transportando ar quente e úmido para o Sul, como também a presença de jato subtropical de oeste em altos níveis. A figura 6 apresenta a síntese dos registros de tornados por estação do ano na área de estudo, no período de 1959 a 2018.

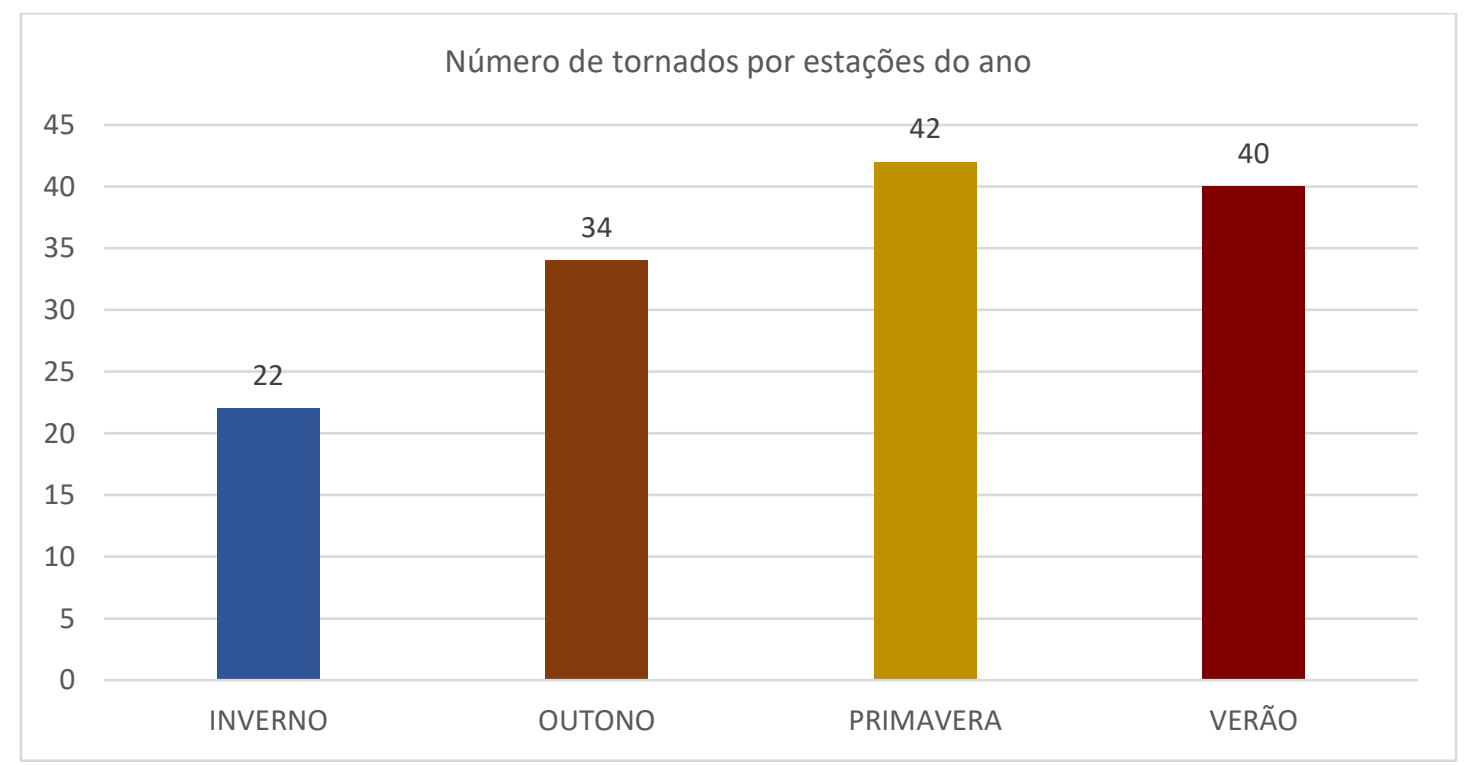

Figura 6. Número total de tornados registrados por estação do ano. Org.: Os autores (2018). 
Diferentemente do que se observa nos Estados Unidos, que tem seu ápice de tornados na primavera, a área de estudo mostrou a partir dos registros que os tornados ocorreram em sua maioria tanto no verão quanto na primavera. As regiões do Sul do Brasil mostram um aumento geral das atividades convectivas durante a primavera, verão e outono, com outubro sendo um mês particularmente ativo para os três.

Ainda, se coloca que a distribuição regional também é consistente com sistemas que começam como tempestades mais isoladas com núcleos convectivos profundos, nesse sentido há propagação para leste enquanto crescem em tempestades contendo núcleos convectivos largos (RASMUSSEN e HOUZE, 2011).

\section{CONCLUSÕES}

Os tornados não são fenômenos raros e podem ocorrem em diversas áreas do Brasil e do mundo. Ao contrário do que se pensava a respeito de tornados serem eventos de difícil ocorrência no Brasil, esse estudo reforça outros pioneiros de que há registros pelo menos desde a década de 1950. Por ser um evento de difícil previsão por sua escala microclimática, antes dos anos de 1990 havia pouquíssimos registros. Por outro lado, com a inovação tecnológica, crescimento da população e a instantânea propagação das notícias a partir de 1990 notou-se o crescimento nos registros de tornados na área de estudo.

A partir dos resultados observados em relação aos sistemas atmosféricos, a Frente Polar Atlântica foi o sistema atmosférico que mais esteve presente nos registros de tornados, observando-se que o domínio dos sistemas extratropicais (massas e correntes perturbadas) ocorreu em $84,1 \%$ dos eventos tornádicos registrados na área de estudo em comparação com os registros sob condições de sistemas de origem intertropical. A análise da sazonalidade mostrou que os eventos ocorrem principalmente na primavera e no verão. 
Ao observar a dinâmica atmosférica regional da área de estudo, notou-se grande semelhança com o Corredor dos Tornados nos Estados Unidos, pois ambas possuem os mesmos fatores climáticos que dão origem aos eventos tornádicos. Em especial, observou-se o ar frio vindo da Patagônia que adentra o continente Sul-americano e encontra-se com o ar quente e seco que se origina na Baixa do Chaco que se origina a leste do Andes pela frontólise da Frente Polar Pacífica que ao extravasar a cordilheira sofre dissecação adiabática.

Observou-se também o ar quente e úmido vindo do oceano originado da Massa Tropical Atlântida além da Corrente do Jato que atua em alto nível da atmosfera se deslocando de oeste para leste colaborando para trocas de energia na atmosfera. Todos os sistemas atmosféricos descritos são elementos que proporcionam a dinâmica atmosférica que eventualmente podem vir a formar tempestades tornádicas.

Com o aumento dos registros de tornados, há também a preocupação de que eles ocorram em áreas habitadas causando vítimas fatais, além de prejuízos. Mesmo com o avanço tecnológico, os tornados ainda não são tratados como uma ameaça potencial como os desastres naturais de inundações e deslizamentos, por exemplo. Nesse sentido, ao realizar essa pesquisa, buscou-se contribuir com a climatologia de tornados e espera-se o surgimento de novas técnicas de previsão e mitigação dos impactos e perdas causadas pelos tornados no Brasil.

\section{REFERÊNCIAS}

BERTONI, B. G. L. Estudos de caso de tornados, uso e ocupação do solo, topografia e análise meteorológica de eventos atmosféricos no sul e sudeste do Brasil. Tese (Doutorado em Ciências Ambientais) - Universidade de São Paulo. São Paulo. 2013.

BREUniG, F. M.; ROBAINA, L. E. S.; BRUM, L.; PAZ, V. P.; PIRES, C. A. F.; MEDEIROS, E. R. O Rio Grande do Sul através do tempo geológico: proposta didática. Revista GeoUERJ. Rio de Janeiro, v. Especial. p. 27-40, 2003.

CANDIDO, D. H. Tornados e trombas d'água no Brasil: desenvolvimento de um modelo e proposta de escala de avaliação de danos. 2012. 230 f. Tese (Doutorado em Geociências) - Universidade Estadual de Campinas. 2012.

CHAUDHURI, S. Data mining and database systems: where is the intersection? Bulletin of the IEEE Computer Society Technical Committee on Data Engineering. v. 21, n. 1, p. 1-5. mar. 1998.

COSSETIN, F.; NUNES, A. B.; TEIXEIRA, M. S. Análise do movimento vertical sob duas configurações de altos níveis da troposfera. Revista Ciência e Natura. v. 38. p. 484-490. 2016. 
FAYYAD, U. M.; PIATESKY-SHAPIRO, G.; SMYTH, P. From data mining to knowledge discovery: an overview. In: FAYYAD, U. M.; PIATETSKY-SHAPIRO, G.; SMYTH, P.; UTHURUSAMY, R. (Ed.) Advances in knowledge discovery in data mining. Menlo Park: AAAI Press/The Mit. Press, 1996, p. 1-34.

GALVANI, E.; AZEVEDO, T. R. A Frente Polar Atlântica e as características de tempo associadas: estudo de caso. Textos do Laboratório de Climatologia e Biogeografia - Departamento de Geografia / FFLCH / USP - Série TA - Texto 018. 2012 .

GLICKMAN, T. S. Glossário de Meteorologia. 2ª Ed. Boston: American Meteorological Society. 855 p. 2000.

GUEDES, R. L.Condições de grande escala associadas a sistemas convectivos de mesoescala sobre a região central da América do Sul.1985. Dissertação (Mestrado em Ciências Atmosféricas), Instituto Astronômico e Geofísico/Universidade de São Paulo, 89p.1985.

HUSCHKE, R. E. Glossary of meteorology. Boston: American Meteorological Society. 1959.

INSTITUTO BRASILEIRO DE GEOGRAFIA E ESTATÍSTICA (IBGE). 2004. Mapa de Biomas do Brasil, primeira aproximação. Rio de Janeiro: IBGE. Disponível em www.ibge.gov.br. Acesso em: 27 out. 2017.

IENSSE, A. C. Circulação atmosférica regional e a ocorrência de tornados e trombas d'água no Rio Grande do Sul, no período de 2001 a 2015. 70p. (Trabalho de Graduação) - Universidade Federal de Santa Maria. 2015.

LIMA, J. S. Ocorrência de uma Nuvem Funil em Santa Maria-RS, In: Congresso Brasileiro de Meteorologia. 2. 1982, Pelotas. Anais... Pelotas: UFPEL, 1982.

MADDOX, R. A. Mesoscale convective complexes. Bulletin of American Meteorological Society. n. 61, p. 1374-1387. 1980.

MASSAMBANI, O.; CARVALHO, L. M. V.; VAZQUEZ, M. A. Tornado ou micro explosão? Um diagnóstico via Radar do evento de Itu - São Paulo. In: Congresso Brasileiro de Meteorologia, 7., 1992, São Paulo. Anais... São Paulo: Sociedade Brasileira de Meteorologia, 1992, p.763-768.

MENDONCA, F.; DANNI-OLIVEIRA, I. M. Climatologia: noções básicas e climas do Brasil. São Paulo: Oficina de Texto, 200\%. 206 p.

NASCIMENTO, E. L. Previsão de tempestades severas utilizando-se parâmetros convectivos e modelos de mesoescala: uma estratégia operacional adotável no Brasil. Revista Brasileira de Meteorologia, São José Dos Campos, v. 20, n. 1, p.121-140, 15 fev. 2005.

NECHET, D. Ocorrência de tornados no Brasil. Boletim da Sociedade Brasileira de Meteorologia, v. 26, n. 2, p. 29-39, dez. 2002.

NIMER, E. Circulação atmosférica do Brasil (Comentários) Contribuição ao estudo da Climatologia do Brasil. Revista Brasileira de Geografia. Rio de Janeiro: IBGE. p. 232 - 250. 1966.

NIMER, E, Clima Região Sul.In: Geografia do Brasil. Rio de Janeiro: IBGE. 2 ed. 1990.

NATIONAL OCEANIC AND ATMOSPHERIC ADMINISTRATION (NOAA). Climate Diagnosties Bulletin. Mariland: NOAA Office for Coastal Management, 1995.

NATIONAL OCEANIC AND ATMOSPHERIC ADMINISTRATION (NOAA). Severe Weather 101 - Tornadoes Basics. Disponível em: https://www.nssl.noaa.gov/education/svrwx101/tornadoes/.Acesso em 25 maio. $2018 \mathrm{a}$. 
NATIONAL OCEANIC AND ATMOSPHERIC ADMINISTRATION (NOAA) - Índice multivariado de EI Niño Oscilação Sul (IME). Earth System Research Laboratory/Physical Science Division/National Oceanic and Atmospheric Administration. Disponível em: http://www.esrl.noaa.gov/psd/enso/mei/, acesso em: 18 de fevereiro de 2018b.

RASMUSSEN K. L.; HOUZE R. A. Jr. Orogenic convection in South America as seen by the TRMM satellite, Monthly Weather Review. Boston. n. 139, p. 2399-2420.2011.

RECKZIEGEL, B. W. Levantamento dos desastres desencadeados por eventos naturais adversos no Estado do Rio Grande do Sul no período de 1980 a 2005. Dissertação (Mestrado em Geografia) - Universidade Federal de Santa Maria, Santa Maria, $2007.370 \mathrm{f}$.

RIBEIRO, A. G. As escalas do clima. Boletim de Geografia Teorética, Rio Claro-SP, v.23, n.45-46, p.288-294, 1993.

RODRIGUES, J. E. A. Cartografia da ocorrência de tornados no Centro-sul do Brasil, no período de 1980 a 2008.

(Trabalho de Graduação). Universidade de São Paulo. São Paulo. 2011.

SARTORI, M. G. B. A dinâmica do clima do Rio Grande do Sul: indução empírica e conhecimento científico. Revista Terra Livre, São Paulo, v. 1, n. 20, p. 27-49, jan./jul. 2003.

SILVA DIAS, M. A. F. e GRAMMELSBACHER, E. A. A possível ocorrência de tornado em São Paulo no dia 26 de abril de 1991: um estudo de caso. Revista Brasileira de Meteorologia, v. 6, n. 2, p. 513-522, 1991.

VELASCO, I. J. M. FRITSCH, J. M.: Mesoscale convective complexes in the America. Journal of Geophysical Research. n. 92, p. 9591-9613. 1987 .

VESILIND, P. J. Chasing tornadoes. National Geographic, p. 2-37, Abr. 2004. 